\title{
Hemimandibulectomy of an extensive complex odontoma in the mandible: a case report
}

\author{
Retno Widayanti ${ }^{1 *}$, Andri Hardianto ${ }^{1}$, Winarno Priyanto ${ }^{2}$, Kiki A. Rizki ${ }^{3}$
}

\section{Abstract}

Objective: Odontomas are the most common form of odontogenic tumors of the jaw. They constitute $22 \%$ of all odontogenic tumors. Odontomas are of two types: a. compound and b. complex. Compound odontomas contain recognizable enamel, dentin, and sometimes cementum, and appear to be tooth-like structures, whereas complex odontomas are composed of irregular masses of dentin and enamel and have no anatomic resemblance to a tooth.

Methods: A17-year-old female patient came to Department of Oral and Maxillofacial Surgery with a slow-growing and asymptomatic swelling in her left mandible. The panoramic radiograph showed a radio-opacity and radiolucent lesion, with well-corticated limits. The radio-opaque area was amorphous, circumscribed by a thin and irregular radiolucent halo. An incisional biopsy confirmed the lesion as a complex odontoma. The surgery performed was hemimandibulectomy followed by a reconstruction procedure using a plate under general anesthesia.

Results: Complex odontomas are most likely present in the posterior region of the maxilla or the mandible. They can be treated with a simple enucleation and curettage. In this case report, hemimandibulectomy was performed due to the extensiveness of the mass.

Conclusion: Surgical removal of large complex odontoma with hemimandibulectomy is a rare clinical scenario. It is only the extensiveness of the lesion that contributed to opting for hemimandibulectomy as the surgical option to remove the lesion.
${ }^{1}$ Department of Oral and Maxillofacial Surgery, Faculty of Dentistry, Padjadjaran University, Bandung, Indonesia

${ }^{2}$ Department of Oral and Maxillofacial Surger, Dr. Hasan Sadikin General Hospital, Bandung, Indonesia.

${ }^{3}$ Department of Head and Neck Oncology, Faculty of Medicine, Padjadjaran University, Dr. Hasan Sadikin General Hospital, Bandung, Indonesia.
*Corresponding to: Retno Widayanti, Department of Oral and Maxillofacial Surgery, Faculty of Dentistry, Padjadjaran University, Bandung, Indonesia

retnowidayanti99@yahoo.co.id

\section{Received: 29 August 2017}

Revised: 9 October 2017

Accepted: 16 October 2017

Available Online: 1 December 2017

Keywords: Complex, Compound, Mandible, Odontoma

Cite this Article: Widayanti R, Hardianto A, Priyanto W, Rizki KA. 2017. Hemimandibulectomy of an extensive complex odontoma in the mandible: A case report. Journal of Dentomaxillofacial Science 2(3): 187-190. D01: 10.15562/jdmfs.v2i3.518

\section{Introduction}

Odontomas are the most odontogenic tumors; however, they are found not as a true odontogenic neoplasm but rather as hamartomas. ${ }^{1,2}$ Odontoma was first described to have been formed by an overgrowth of complete dental tissue. ${ }^{2}$ Odontoma is a benign odontogenic tumor that is composed of dental tissues: enamel, dentin, and cementum. ${ }^{2,3}$

The second edition of the WHO histologic typing of odontogenic tumors classifies odontomas under the broad category of tumors containing odontogenic epithelium with odontogenic ectomesenchyme, with or without dental hard tissue formation, and based on this classification, three specific types of odontomas have been identified: odontoameloblastoma, complex odontoma, and compound odontoma. ${ }^{2}$ According to WHO classification of odontogenic tumors as published in 2005, odontomas are basically classified into compound and complex odontomas. ${ }^{1-3}$ Compound odontoma is characterized by normal tooth-like elements such as dentin, enamel matrix, cementum, and pulp tissue; on the other hand, complex odontomas are characterized by tumor-like malformation or hamartomas, in which the enamel, dentin, and sometimes cementum are present as irregular calcified mass without normal tooth-like anatomy. ${ }^{1,4,5}$ Odontomas have also been classified as central, peripheral, and erupted odontomas according to the symptoms identified during clinical presentation. $^{2}$

In $22 \%$ of the cases, odontogenic tumors of the jaws are identified as odontoma. ${ }^{2,3}$ Compound odontomas are usually located in the anterior maxilla and complex odontomas are usually located in the posterior of mandible and posterior maxilla., ${ }^{4,5}$ Complex odontomas occur less frequently than compound odontoma, in the ratio of $1: 2 .^{2,5}$

Odontoma can occur in both males and females, but it occurs more frequently in woman and usually before the second decade of life. ${ }^{4}$ Odontoma grows asymptomatically; it grows slowly, non-aggressively, and reaches no more than $3 \mathrm{~cm}$ in diameter. ${ }^{4,5}$

This article describes a rare case of complex and rather large-size odontoma, measuring more than $3 \mathrm{~cm}$ in diameter, in the left posterior of mandible along with thinning of left cortical mandible bone and impacted teeth. The lesion was removed with hemimandibulectomy.

\section{Case Report}

A 17-year-old girl came to Oral and Maxillofacial Department of Dr. Hasan Sadikin General Hospital; the symptoms she presented included swelling of 
her left lower jaw, which she said she had for the last 4 years. The swelling initially recognized in her left gum with hard consistency and asymptomatic with slow growth. There was no history of inflammation or dental or maxillofacial trauma in her left lower jaw. The patient also had no problem with chewing or inability in opening or closing her mouth. The patient did not have history of decreasing body weight recently and had no other swelling in her body. No similarity or history of other swelling in her family.

From general examination, the symptoms appeared normal. From local extra oral examination it was found that her face was asymmetrical due to swelling in her left mandible with skin color similar to the surrounding skin and mucosa figure 1 . With palpation from extra-oral, we found there was a mass at left mandible, which was afebrile, showed hard consistency, had an un-palpable edge of mass, appeared localized, non-tender, had uneven surface, with size measuring $8 \times 6 \times 4 \mathrm{~cm}$. Lymph nodes were not palpable.

Intraoral examination see figure 2, revealed the absence of teeth of 36,37 and 38 with persistence of
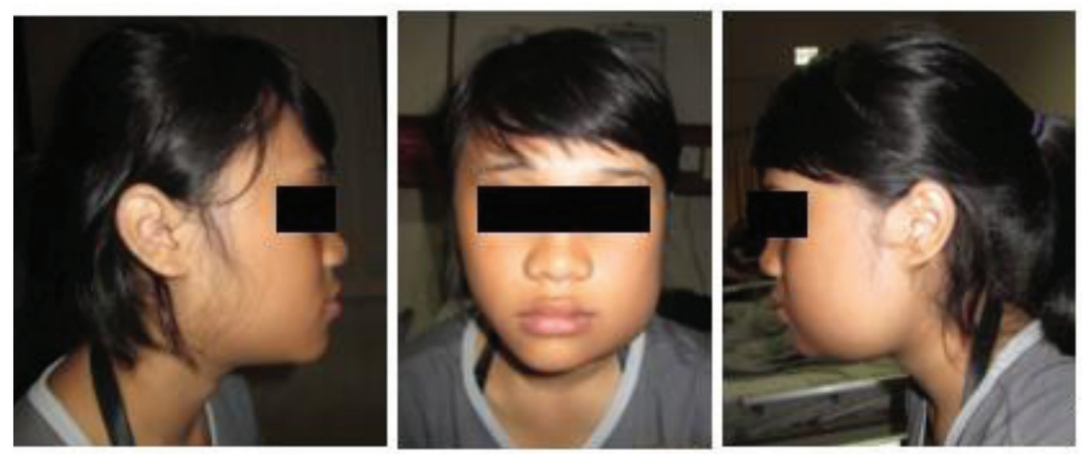

Figure 1 Profile preview before surgery

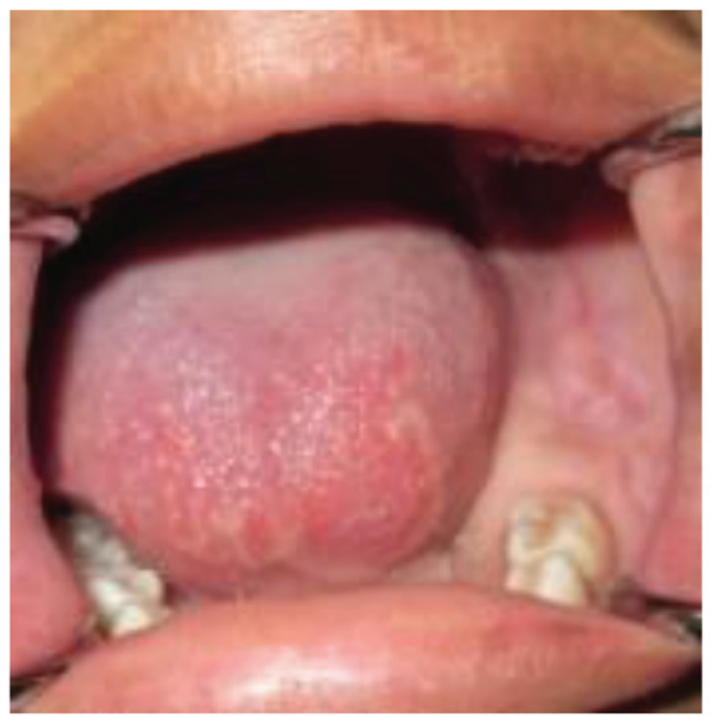

Figure 2 Intraoral view

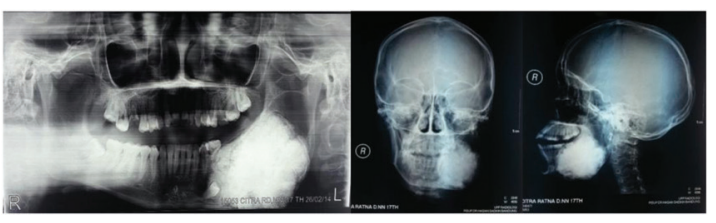

Figure 3 Orthophantomogram, anteroposterior and lateral skull radiograph view

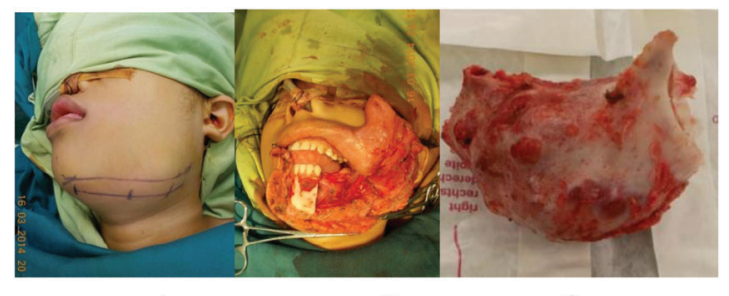

A

B

$\mathrm{C}$

Figure 4 Durante op, A. Design of incision with methylene blue, B. Hemimandibulectomy of left mandible distal to tooth 33, C. Mass of complex odontoma

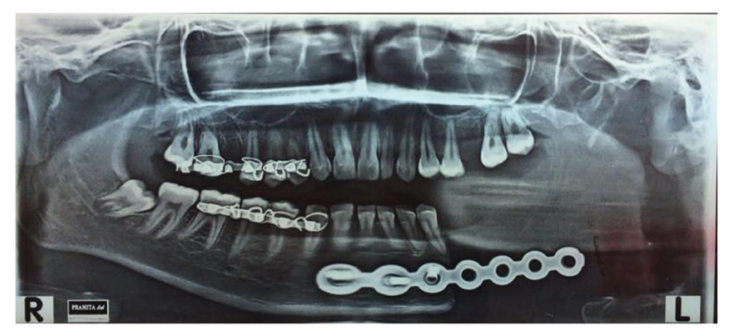

Figure 5 Panoramic radiograph 3 months post-surgery

tooth 75 , and the color of the surrounding mucosa appeared to be similar to the one without ulcers. On palpation, we found that the mass had hard consistency, was localized with uneven surface, had an unclear edge of mass, appeared non-tender and was immobile.

The patient was first diagnosed with tumor of mandible suspect odontoma, ossifying fibroma and ameloblastoma. Incisional biopsy was planned to perform under general anesthesia after taking chest, skull, and panoramic radiograph along with complete blood examination and consultation with anesthesia department.

From odontophantomograph and anteroposterior and lateral view of the skull, a radio-opaque circumscribed mass with irregular shape of left mandible with size measuring $8 \times 6 \times 4 \mathrm{~cm}$ was found. The mass has an extensive size from corpus, angulus, and ramus of the left mandible, and there was an impact of tooth 35 which forced itself into inferior. The unspecified radio-opaque mass was covered with a thin layer of irregular radiolucent. Radiolucent view of clear and unequivocal 
boundary extends to the notch of mandible with thin margins of left mandible ramus figure 3.

After completing the diagnosis, including clinical, radiograph, and histopathological examinations, we concluded that the patient had a complex odontoma in the left mandible and opted for hemimandibulectomy. Surgery was initiated with incisional pattern on the left mandible with methylene blue figure 4. Furthermore, using blade No. 15, we made a transverse incision in the left sub-mandible region, sub-mental, and up to medial lip and cauterized the incision and blood vessels with ligation of vein and cut the mandible form distal to tooth 33 . Removal was made up until the point of coronoid bone and mandibular condyle of the left mandible. Plating and screwing was then used to reconstruct the left mandible. Bandage pressure was applied in the post-incision region, and the patient was instructed to have a liquid diet through nasogastric tube or NGT.

Three months after surgery, patient had a panoramic $\mathrm{x}$-ray figure 5 and reported that she had no pain and swelling and we did not any signs of infection. Patient was then referred to prosthodontic department for prosthesis.

\section{Discussion}

Odontoma is an odontogenic tumor that occurs very frequently, and some of the tumors are termed hamartoma, not as a true tumor. Generally, odontoma grows asymptomatically and is detected only accidentally during routine procedures like radiographic examination when the dentist tries to find the possible causes of delayed dentition or there is a deciduous tooth persistence..$^{3-5}$

In some cases of odontomas, signs will include persistence of deciduous teeth, un-erupted permanent teeth, swelling, pain, suppuration, expansion of cortical bone and shifting of tooth due to pressure of lesions. Other signs of odontoma can be tooth root resorption above the lesions, typically identified during paresthesia, or anesthesia on lower lip. ${ }^{2,3,5}$ In this case, there was a persistence of tooth 75 with the impact of tooth 35 that was shifting due to odontoma lesion.

Most odontomas manifest as a small lesion as intraosseous, calcified, odontogenic tissue., ${ }^{3,4}$ Growth of odontoma was achieved through the stages of odontogenic mineralized tissue reaching a mature stage when it became fully calcified. In the mature stage there may not be occurrences of episodes of new growth, so odontomas are known as lesions that are not aggressive with limited potential growth. ${ }^{4}$
The etiology and pathogenesis of odontoma is not known for certain. Some say that odontoma exists due to local trauma during deciduous teeth, infection, hereditary factor and genetic mutation. ${ }^{2,3,5}$ Odontoma in hereditary anomaly can occur as a manifestation of some syndromes, including gardner syndrome, Hermann syndrome and basal cell nevus syndromes. ${ }^{3,5}$

In this case report the patient was a 17 -year-old female and the odontoma was found in her left mandible. This is consistent with the theory that complex odontomas are usually found in the posterior region of mandible., ${ }^{2,5}$ Complex odontoma occurs less frequently than compound odontoma, in the ratio of $1: 2.2,5$

Differential diagnosis of odontoma is cementoblastoma, osteoid osteoma and fibro-osseus lesions like cemento-ossifying fibroma. ${ }^{3,5}$ On radiological examination, cementoblastoma appeared as radioopaque mass demarcated, attached to the apical tooth and surrounded by radiolucent edge. Osteoid osteoma has a characteristic picture of ovoid radiolucency and is surrounded by sclerotic bone boundary; the central radiolucent with increased calcification spots without surrounding radiolucent boundary can lead physicians to suspect it is a normal bone. ${ }^{5}$

Radiography showed there are three different development stages in odontoma, depending on the level of odontoma calcification. In the initial stage it appears like a radiolucent lesion without calcification, and at intermediate stages the lesions appear partially calcified and in the final stage of odontoma, the lesion become radio-opaque and appears surrounded by a radiolucent halo. ${ }^{2}$ Microscopic examination should be done in both types of odontoma. ${ }^{2,4}$ Histologist's examination should be done carefully, especially when dealing with extensive dimensions of lesion because it can be misdiagnosed for other odontogenic tumors, which have the potential to grow broader and are expansive and more aggressive, such as odontoma fibro ameloblastoma, odontoameloblastoma and calcified odontocystic tumor.

Surgical management of odontoma removal is by excisional surgery. In this case report, the odontoma has a large size and its extensive lesion has caused damage to surrounding structures, accompanied by impaction and thinning of mandibular cortical bone. This fact shows that the mass of odontoma has a rhythm of growth that cannot be predicted and the lack of differentiation of odontogenic epithelial in complex variant. ${ }^{4}$ Because of the huge and expanding mass of the lesion, radical surgery of hemimandibulectomy on the left mandibular region was performed. 
Mass of odontoma if discovered early when it is still small can be removed by enucleation and curettage; in this case report it could not have been done because the mass located was large and extensive. Although in the case of odontoma the likelihood of recurrence is rare, there may be other issues, especially in this case, such as defects arising after hemimandibulectomy action that would be a problem in themselves considering the patient is still young.

\section{Conclusion}

Odontoma is an odontogenic tumor that most usually occurs in the jaw and can be found by the dentist during routine examination along with panoramic radiograph. Finding an extensive odontoma that needs radical surgery for removal is a very rare case. Early diagnosis and appropriate management should be done to prevent any complications.

\section{Conflict of Interest}

The authors report no conflict of interest.

\section{References}

1. Bagheri SC, Bell RB, Khan HA. Current therapy in oral and maxillofacial surgery. St. Louis Missouri: Elsevier saunders; 2012. p. 402-405.

2. Patil S, Rahman F, Tipu SR, et al. Odontoma: review of literature and report of a case. J Oral Maxillofac Pathol 2012;3: 224-227.

3. Krichen G. Hentati H, Hadhri R, et al. Odontoma associated with supernumerary and impacted teeth. Int Dent J Students Res 2013;1: 47-52.

4. Pedro HRS, Tulio HS, Servato JP, et al. Giant complex odontoma of the anterior mandible: Repost of case with long follow up. Br Dent J 2012;23: 597-600.

5. Vengal M, Arora $\mathrm{H}$, Ghosh $\mathrm{S}$, et al. Large erupting complex odontoma: a case report. J Can Dent Assoc 2007;73: 169-172.

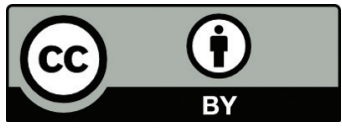

This work is licensed under a Creative Commons Attribution 